\title{
Targeted Ultrasound-Guided Double Catheters (Infraclavicular-Brachial Plexus, Median Nerve) Facilitate Hand Rehabilitation with Superb Analgesia and Motor Function Retention
}

\author{
Ashlee E. Holman, Balram Sharma, Vicki E. Modest \\ Massachusetts General Hospital, Department of Anesthesia, Critical Care, and Pain Medicine, Boston, \\ USA \\ Email: ashlee.holman@gmail.com
}

Received 8 June 2015; accepted 19 July 2015; published 22 July 2015

Copyright (C) 2015 by authors and Scientific Research Publishing Inc.

This work is licensed under the Creative Commons Attribution International License (CC BY). http://creativecommons.org/licenses/by/4.0/

(c) (i) Open Access

\section{Abstract}

A 44-year-old male who suffered a crush-degloving hand injury complicated by Complex Regional Pain Syndrome (CRPS) type I was scheduled for operative hand manipulation and inpatient physiotherapy. Preoperative placement of an ultrasound-guided infraclavicular catheter provided incomplete analgesia requiring supplemental morphine during physiotherapy sessions despite continuous infusion of $0.1 \%$ bupivacaine at $20 \mathrm{~mL} /$ hour. Due to the patient's adamant refusal of replacement of the infraclavicular catheter, a second ultrasound-guided median nerve catheter was placed distally at the mid-forearm level and elicited complete sensory blockade of the hand. Dual infusions were maintained with $0.25 \%$ bupivacaine at $5 \mathrm{~mL} /$ hour through the median nerve catheter and $0.1 \%$ bupivacaine at $20 \mathrm{~mL} /$ hour through the infraclavicular catheter, and subsequent daily physiotherapy progressed productively. Continuous perineural catheter use to facilitate rehabilitation is an emerging practice that may improve overall recovery. Combination catheters, infusing local anesthetics at separate locations, can be used synergistically to preserve motor function and reach a superior analgesic endpoint.

\section{Keywords}

Double Perineural Catheters, Infraclavicular Catheter, Median Nerve Catheter, Selective Sensory Blockade, Motor Function Preservation 


\section{Introduction}

This medically challenging case is the first report in the literature describing the use of combined infraclavicular and distal mid-forearm median nerve catheters to provide analgesia specifically with the goal of enabling physiotherapy in a patient who has failed hand rehabilitation as a result of uncontrollable pain due to CRPS type I.

Sensory blockade of an injured hand, with preservation of motor function, is ideal during hand therapy; however, this goal may be elusive. The effect of local anesthetic concentration and total volume on nerve blockade is complex and may vary with concentration, total dose, and location. In this case, each catheter was infused with different concentrations of local anesthetic to achieve specific treatment goals. The "failed" brachial plexus catheter was re-purposed to provide generalized analgesia for the injured limb via the infraclavicular route, and in the most painful area of the hand, a denser block was obtained via addition of the median nerve catheter [1].

\section{Case Presentation}

A 44-year-old male (190 cm, $93 \mathrm{~kg}$, ASA 1) suffered a crush-degloving injury to his dominant hand requiring extensive emergency surgery at an outside hospital. The neurovascular and tendon structures were surgically repaired without complication. Early rehabilitation was initiated but progressively declined due to the development of CRPS type I in the first five months post repair. His symptoms included severe resting hyperalgesia and allodynia in his hand and wrist with spread to his upper arm as well as dermatological changes such as swelling, erythema, and hyperthermia.

At post-operative week 8, passive range of motion was limited to 15-degree flexion at the metacarpal and proximal inter-phalangeal joints secondary to pain and dense scar tissue. At 22 weeks post-injury, he was readmitted for operative digital manipulation and five days of inpatient occupational therapy.

\subsection{Pre/Intraoperative Course}

Intraoperative digital manipulation was preceded by uneventful infraclavicular brachial plexus peripheral nerve catheter placement using an in-plane technique under continuous ultrasound-guidance (linear $12 \mathrm{MHz}$ transducer, GE Healthcare, Wauwatosa, WI). A 20-gauge Arrow StimuCath catheter was placed through an 18-gauge insulated block needle. Twenty $\mathrm{mL}$ of $0.5 \%$ bupivacaine administered through both the needle and catheter resulted in partial but incomplete brachial plexus blockade. At this time, the patient described pain relief throughout his limb at rest only. Physical manipulation of the hand and wrist elicited pain; however, it was improved from the patient's baseline pain level. Because of elevated patient anxiety due to claustrophobia resulting from sterile drape placement, his refusal of further catheter testing, and his refusal of catheter replacement, operative hand manipulation was performed under combined nerve block and general anesthesia. Infusion of $10 \mathrm{~mL} / \mathrm{hour}$ of $0.1 \%$ bupivacaine through the catheter was initiated in the recovery room, and the patient was comfortable until early on post-operative day (POD) 1.

\subsection{Postoperative Course}

On POD 1, intravenous morphine $53 \mathrm{mg} / 24$ hours and continuous infusion of $20 \mathrm{~mL} /$ hour of $0.1 \%$ bupivacaine administered through the infraclavicular catheter allowed the patient to participate in physiotherapy with a pain Numerical Rating Score (NRS) of 6/10. Pain persisted in the medial aspect of his forearm and diffusely throughout his hand.

On POD 2, despite intravenous morphine $93 \mathrm{mg} / 24$ hours and continuous $0.1 \%$ bupivacaine infusion at 20 $\mathrm{mL} /$ hour, the patient refused physiotherapy. He then presented to us in the pre-operative area for catheter replacement.

On evaluation, administration of $10 \mathrm{~mL}$ of $2 \%$ lidocaine with epinephrine $(1: 400,000)$ through the infraclavicular catheter resulted in improved pain relief; yet, the patient continued with aggressive hand guarding. Despite difficulty in assessment due to patient anxiety and reluctance to participate in sensory testing, it appeared that the focus of pain was primarily across the palmar crease and proximal palmar surface of the hand. Although the palmar crease has the potential to have shared nerve innervation, it seemed that the infraclavicular catheter least covered the median nerve territory. As a result of his claustrophobia and apprehension regarding placement of the sterile draping near his face, the patient adamantly refused replacement of the infraclavicular catheter. We opted to place a second catheter to specifically anesthetize the median nerve, thus providing a less anxiety pro- 
voking environment for the patient as well as specifically targeting sensation without disturbing motor function. Using the same ultrasound machine, the forearm was scanned to visualize the median nerve, and a catheter was placed at the mid-forearm level [2]. See Figure 1 and Figure 2.

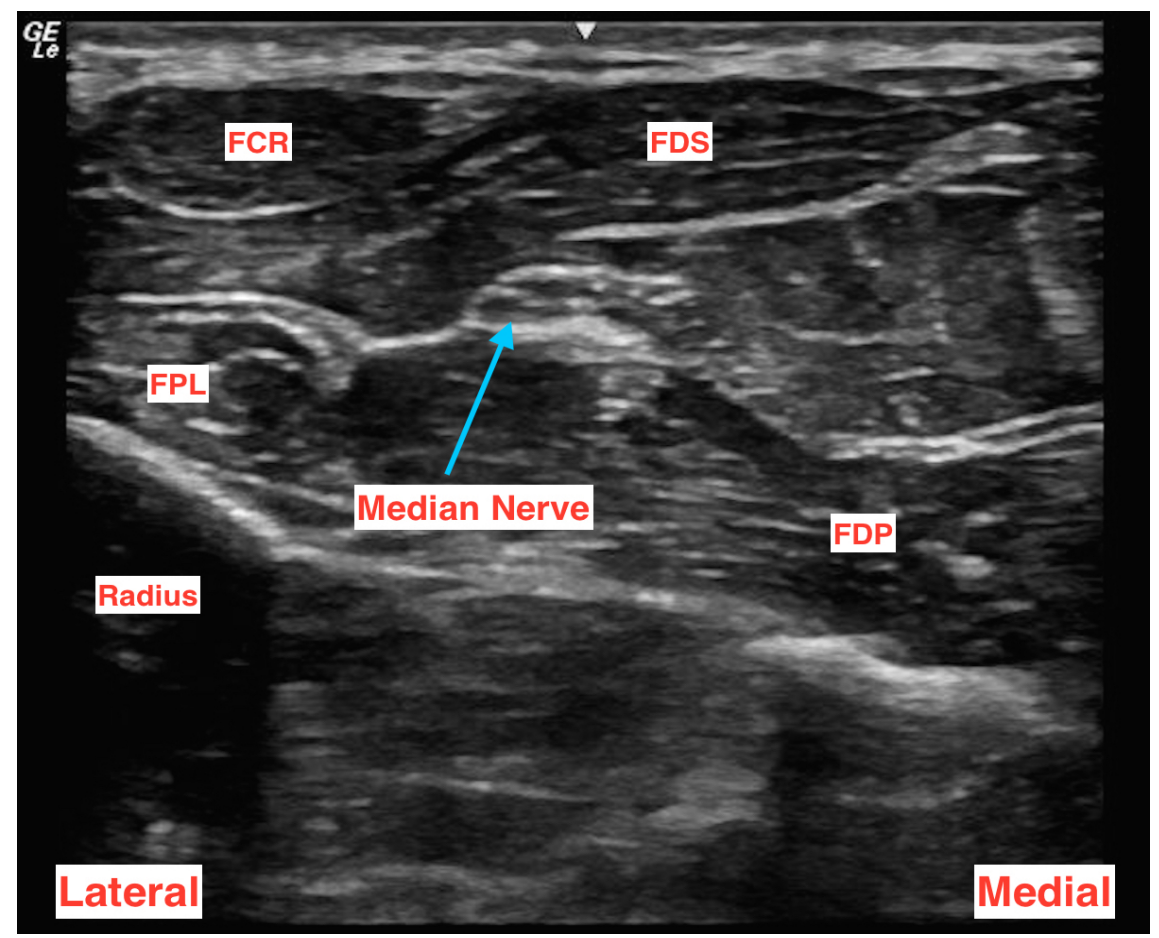

Figure 1. Ultrasound image of the right median nerve at mid-forearm surrounded by the forearm muscle group. a. FCR: flexor carpi radialis; FDP: flexor digitorum profundus; FDS: flexor digitorum superficialis; FPL: flexor pollicus longus.

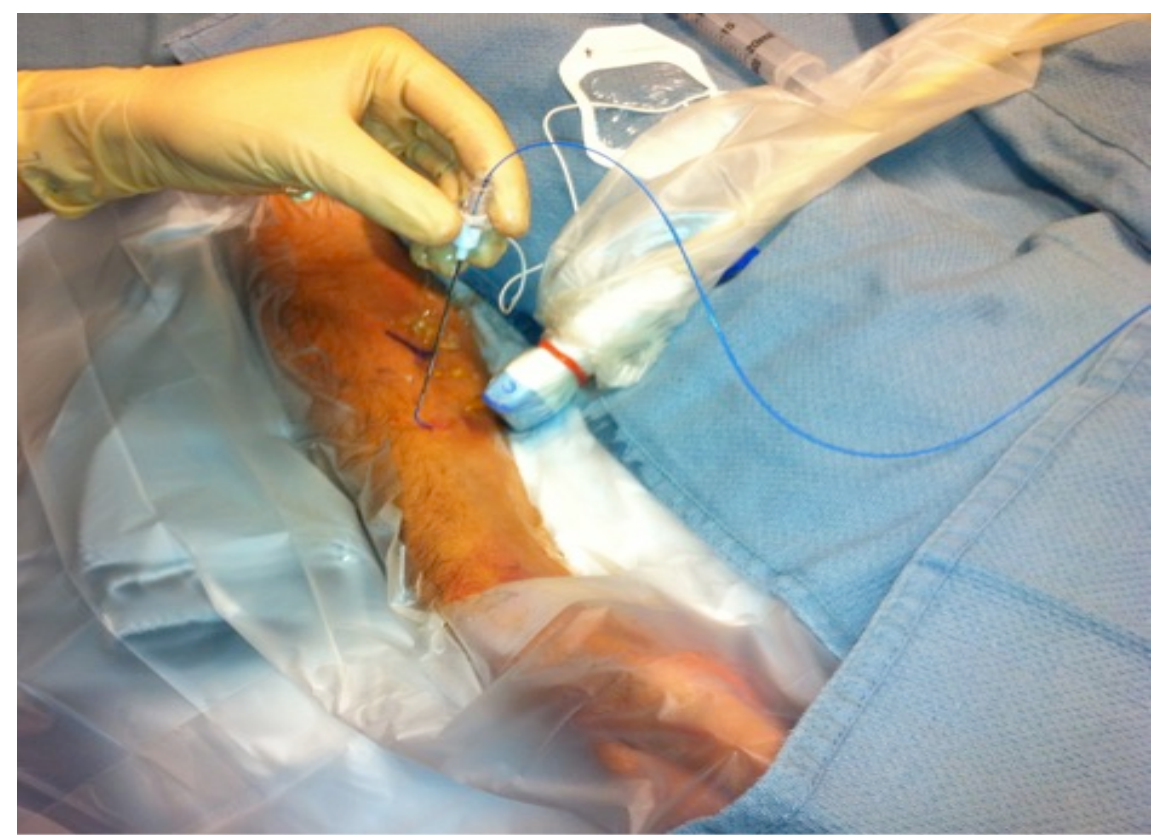

Figure 2. A lateral-to-medial approach was used with in-plane, real-time ultrasound guidance to place the distal median nerve catheter after the AIN branch point. a. AIN: anterior interosseous nerve. 
Five $\mathrm{mL}$ of $2 \%$ lidocaine with epinephrine (1:400,000), administered through the median nerve catheter, provided an aggregate complete sensory blockade of the hand. The patient was able to actively and passively manipulate his hand, pain-free, within 15 minutes. Using a second pump, an infusion of $5 \mathrm{~mL} / \mathrm{hour}$ of $0.25 \%$ bupivacaine was initiated. The infraclavicular catheter infusion was continued at $20 \mathrm{~mL} /$ hour of $0.1 \%$ bupivacaine. See Figure 3.

On POD 3 through POD 5, the patient consistently reported an NRS score of 0/10 and enthusiastically participated in multiple daily physiotherapy sessions. He required minimal opioids. See Figure 4 and Table 1. On

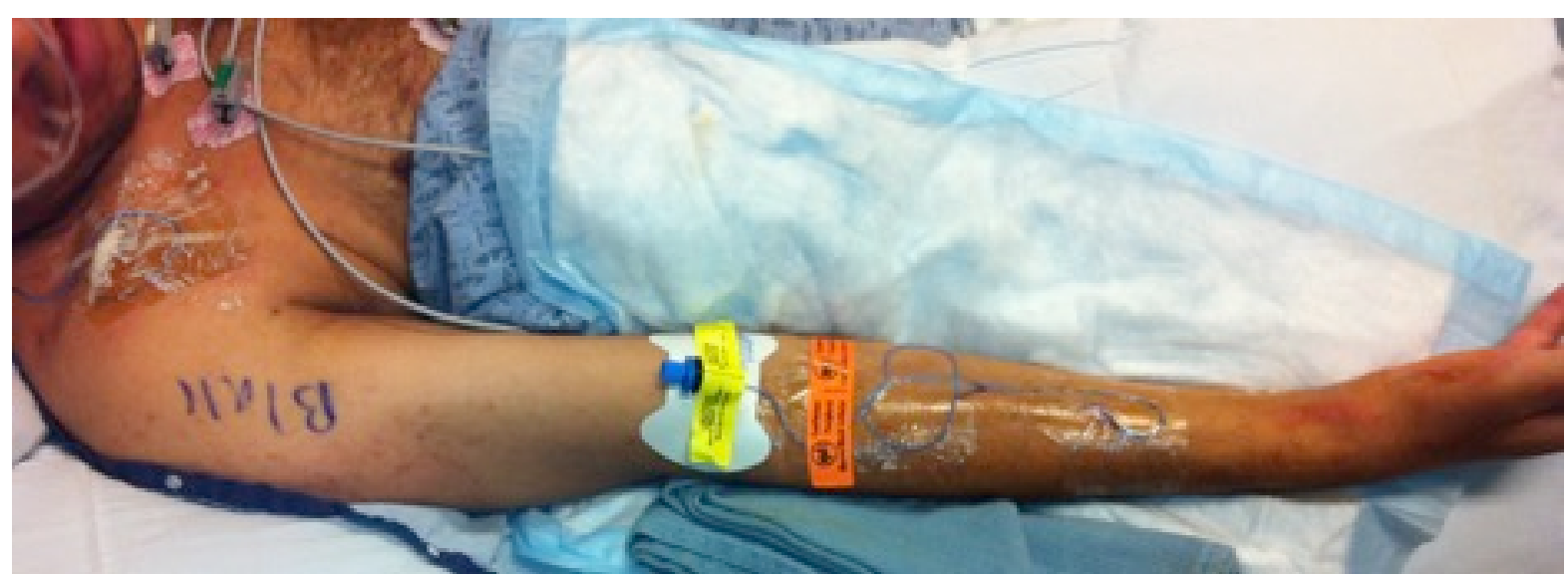

Figure 3. Two dressed and functional catheters: 1) brachial plexus at the infraclavicular level, 2) distal median nerve catheter placed after the AIN branch point. a. AIN: anterior interosseous nerve.

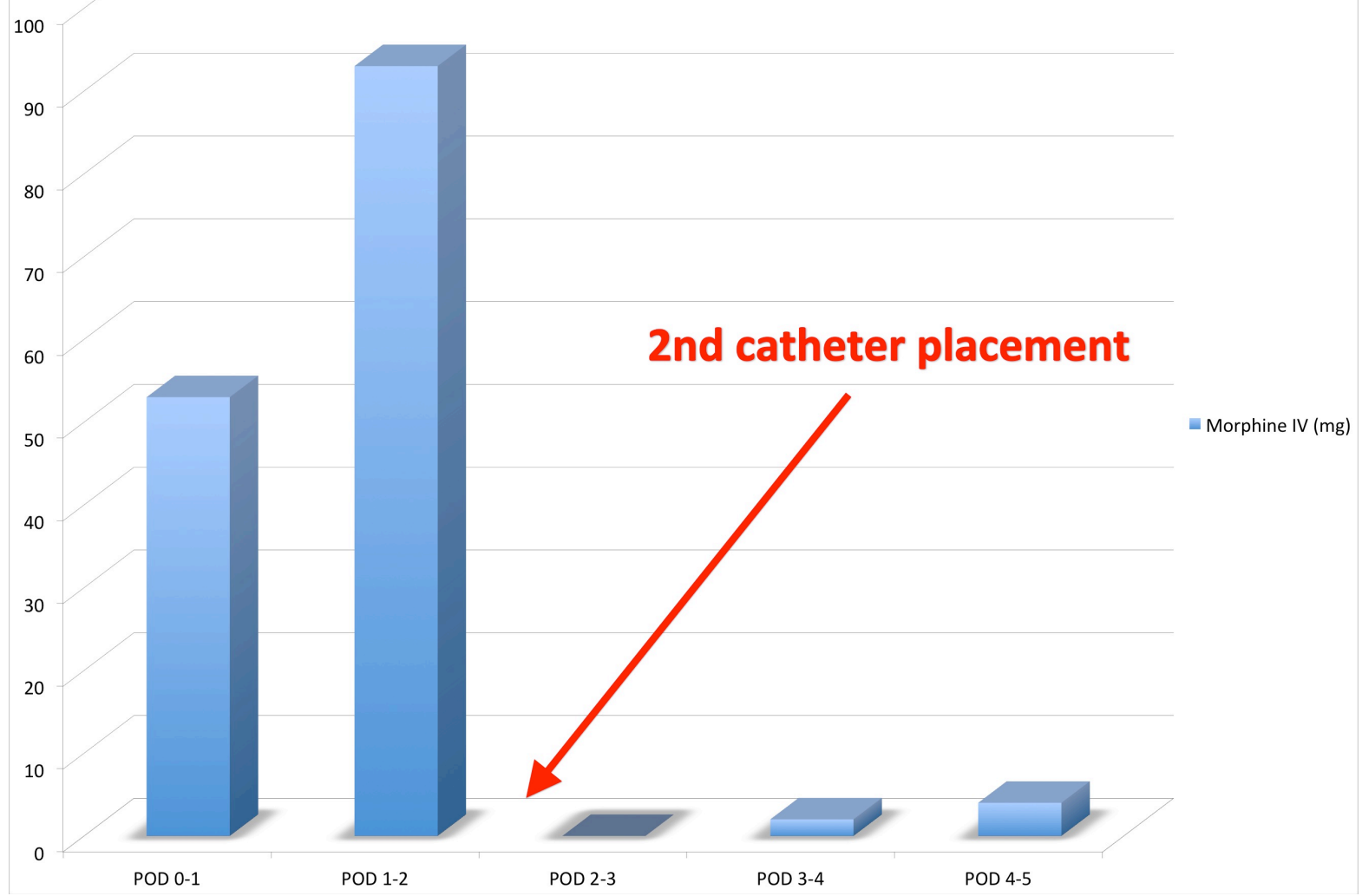

Figure 4. Morphine consumption over 5-day period. Note the insertion of the median nerve catheter on POD 2. a. POD: post-operative day. 
Table 1. Daily morphine (IV) consumption over 5-day period.

\begin{tabular}{cccccc}
\hline & POD $0-1$ & POD $1-2\left(2^{\text {nd }}\right.$ catheter $)$ & POD $2-3$ & POD $3-4$ & POD $4-5$ \\
\hline Morphine IV $(\mathrm{mg})$ & 53 & 93 & 0 & 2 & 4 \\
\hline
\end{tabular}

a. IV: intravenous; mg: milligram; POD: post-operative day.

POD 5, he actively achieved 30-degree flexion and improved extension of all five digits. He was discharged for outpatient physical therapy on POD 6.

\section{Discussion}

We present a medically challenging case reporting the first use of combined infraclavicular and distal median peripheral nerve catheters to allow for intraoperative digital manipulation and rehabilitation of a severely injured and painful hand.

With a favorable risk/benefit profile, single-shot regional blocks provide nearly perfect pain control for up to 24 hours and are routinely used for many surgeries, including upper extremity and hand procedures [3]. For rehabilitation cases, however, peripheral nerve catheters may be preferred as they can extend analgesia for the life of the catheter and allow local anesthetic dose adjustments. This case report illustrates a surgical rehabilitation treatment strategy contingent on adequate catheter functioning.

For our patient, the infraclavicular catheter alone relieved pain only at rest. By the end of POD 1, attempts at hand mobilization, both passive and active, resulted in high pain scores and participation dropout. Infraclavicular catheter evaluation revealed a relatively functional catheter with apparent sparing of the median nerve. Distal nerve branch sparing such as this is not uncommon and, as was true here, may disrupt rehabilitation plans and cause both emotional and physical stress for the patient. Due to his high level of anxiety, claustrophobia, and partial but incomplete pain relief, our patient adamantly refused replacement of the infraclavicular catheter but was amenable to the placement of a second, more distal catheter, which he considered to be a less stressful alternative. Accordingly, a dilute local anesthetic infusion was continued through the first catheter and a second peripheral nerve catheter was placed to target the distal median nerve.

The goal of the second catheter was to provide adequately dense sensory blockade while preserving motor function, thus allowing for active participation in rehabilitation exercises. For the median nerve, selective sensory blockade may be achieved with catheter placement distal to the branching point of the anterior interosseous nerve (AIN), which is responsible for innervation of the 2.5 muscles (flexor pollicus longus (FPL), pronator quadratus, and the radial portion of the flexor digitorum profundus (FDP)) of the deep anterior compartment of the forearm. Normally, the AIN branches from the median nerve within the proximal 1/3 of the forearm [4]-[6].

Ultrasound was used to locate the median nerve at the mid-forearm level. This site was chosen to ensure that the catheter would be placed adequately distal to the branching point of the AIN (to minimize potential motor blockade due to retrograde flow of local anesthetic to the AIN) but before the palmar cutaneous branch of the median nerve [7]. The nerve was identified between the FPL and FDP muscles, adjacent to the palmaris longus tendon. The palmaris longus tendon can be differentiated from the median nerve by having the patient flex his/her wrist, resulting in movement of the tendon but not the nerve [5] [6].

After dual catheter placement, the hand surgeon and physical therapists noted excellent analgesia exemplified by reduced intravenous morphine consumption from $146 \mathrm{mg}$ over 48 hours pre-addition of the median nerve catheter to $6 \mathrm{mg}$ over the subsequent 96 hours. This allowed the patient to comfortably and successfully meet his in-house physical therapy metrics. A plan for repeat inpatient rehabilitation for our patient is currently underway.

Although there are case reports of end-branch peripheral nerve catheter use for hand surgeries and rehabilitation and some reports of multiple catheter use, to our knowledge, this is a novel use of two simultaneous catheters administering different local anesthetic solutions to achieve successful, multi-day hand physiotherapy [7]. Surprisingly, there are few reports of forearm peripheral nerve catheters placed under ultrasound-guidance. We support the use of ultrasound for its ease in aiding in rapid definition of the anatomy, ability to increase accuracy in deposition of local anesthetic, and capacity to allow for close approximation of the catheter to the nerve, permitting the use of low-volume, dilute local anesthetic solution to decrease the risk of neurotoxicity as well as local anesthetic systemic toxicity (LAST). 
Selective sensory blockade can be applied to ulnar and radial nerve catheters as well. Blocking the ulnar nerve distal to the motor branches which supply the flexor carpi ulnaris and the ulnar half of the FDP but proximal to the palmar and dorsal branches of the ulnar nerve allows for selective sensory blockade. Selective blocking of the sensory component of the radial nerve can be done near the wrist, as the deep branch of the radial nerve, which innervates the muscles of the forearm and hand, separates in the distal forearm [8].

Dual catheter therapy should be considered when proximal peripheral nerve catheters have unintentional distal nerve sparing or when disparate sensory blockade densities are needed. Targeted second catheter augmentation may be an effective and easy solution.

Notably, the use of multiple peripheral nerve catheters presents a unique situation in which certain risks of regional anesthesia should be highlighted. Of these, LAST remains the most feared complication of regional anesthesia. For our patient, the use of a dilute solution of local anesthetic through the infraclavicular catheter was employed to decrease the risk of reaching toxic systemic bupivacaine levels from two separate local anesthetic infusions. We advocate patient-specific dose adjustments, heightened vigilance, and prompt treatment at the earliest signs of systemic toxicity in alignment with the LAST treatment algorithm [9].

Peripheral nerve injury is another rare complication of regional anesthesia, with an incidence estimated to be $<1.0 \%$. Permanent injury is even rarer, with an incidence of $0.01 \%$. In theory, placement of a second catheter into a region of a partially blocked extremity could have resulted in nerve damage; however, our patient had intact sensation over the median nerve distribution, thus providing reassurance in our decision to place the second median nerve catheter. Our patient voiced his understanding of the possible risks associated with placement of a second, more distal catheter, and he was willing to accept the theoretical and small risk of nerve damage to avoid a claustrophobic, anxiety-provoking situation in which a sterile drape was placed near his face. He strongly supported an intervention that could facilitate successful physiotherapy and improved rehabilitation outcome. In addition, our use of ultrasound for catheter placement may have increased the overall safety of the procedure; however, at this time, there are no trials that can definitely support this statement [9].

\section{Conclusion}

In conclusion, the use of continuous peripheral nerve catheters for enablement of physical therapy and rehabilitation is an emerging practice. Selective sensory blockade may be the preferable catheter-related outcome by both patient and physical therapy standards and can be obtained with distal placement of specific nerve catheters. Combination catheters, infusing different local anesthetic solutions at separate locations, can be used synergistically to reach a superior endpoint.

\section{References}

[1] Ilfeld, B.M., Le, L.T., Ramjohn, J., Loland, V.J., Wadhwa, A.N., Gerancher, J.C., Renehan, E.M., Sessler, D.I., Shuster, J.J., Theriaque, D.W., Maldonado, R.C., Mariano, E.R. and PAINfRETM Investigators (2009) The Effects of Local Anesthetic Concentration and Dose on Continuous Infraclavicular Nerve Blocks: A Multicenter, Randomized, Observer-Masked, Controlled Study. Anesthesia \& Analgesia, 108, 345-350.

[2] Maxwell, B.G., Hansen, J.A., Talley, J., Curtin, C.M. and Mariano, E.R. (2013) Ultrasound-Guided Continuous Median Nerve Block to Facilitate Intensive Hand Rehabilitation. The Clinical Journal of Pain, 29, 86-88. http://dx.doi.org/10.1097/AJP.0b013e318246d1ca

[3] Neal, J.M. and Chan, V.W. (2007) Ultrasound and Regional Anesthesia: A New Section in Regional Anesthesia and Pain Medicine. Regional Anesthesia and Pain Medicine, 32, 373-374.

[4] Watanabe, T., Watanabe, I., Koizumi, M., Petrenko, A.B. and Baba, H. (2012) Alternative Site for Median Nerve Blockade Allowing Early Functional Rehabilitation after Hand Surgery. Canadian Journal of Anesthesia, 59, 58-62. http://dx.doi.org/10.1007/s12630-011-9613-4

[5] McCartney, C.J., Xu, D., Constantinescu, C., Abbas, S. and Chan, V.W. (2007) Ultrasound Examination of Peripheral Nerves in the Forearm. Regional Anesthesia and Pain Medicine, 32, 434-439.

[6] Sunderland, S. (1978) Nerves and Nerve Injuries. 2nd Edition, Churchill Livingstone Inc., Edinburg London and New York.

[7] Lurf, M. and Leixnering, M. (2009) Ultrasound-Guided Ulnar Nerve Catheter Placement in the Forearm for Postopera- 
tive Pain Relief and Physiotherapy. Acta Anaesthesiologica Scandinavica, 53, 261-263.

http://dx.doi.org/10.1111/j.1399-6576.2008.01762.x

[8] Fredrickson, M.J., Ting, F.S., Chinchanwala, S. and Boland, M.R. (2011) Concomitant Infraclavicular plus Distal Median, Radial, and Ulnar Nerve Blockade Accelerates Upper Extremity Anaesthesia and Improves Block Consistency Compared with Infraclavicular Block Alone. British Journal of Anaesthesia, 107, 236-242. http://dx.doi.org/10.1093/bja/aer101

[9] Jeng, C.L., Torrillo, T.M. and Rosenblatt, M.A. (2010) Complications of Peripheral Nerve Blocks. British Journal of Anaesthesia, 105, i97-i107. http://dx.doi.org/10.1093/bja/aeq273 